\title{
Síndrome de Wunderlich, uma causa rara de dor abdominal
}

\author{
Wunderlich Syndrome, a rare cause of abdominal pain
}

\author{
Pedro Oliveira' ${ }^{1}$, Paulo Torres-Ramalho², Luis Lopes ${ }^{2}$ \\ ${ }^{1}$ Serviço de Medicina Interna - Centro Hospitalar de Vila Nova de Gaia/Espinho. ${ }^{2}$ Serviço de Medicina Intensiva - Centro Hospitalar de São João Borrar
}

\begin{abstract}
RESUMO
A síndrome de Wunderlich é uma doença rara que se caracteriza por hemorragia espontânea nos espaços subcapsular e perirrenal. Apresentamos dois casos de síndrome de Wunderlich. Uma mulher de 38 anos com esclerose tuberosa que se apresentou com dor abdominal e hipotensão. A tomografia computadorizada (TC) revelou um hematoma peri-renal. Uma mulher de 86 anos, anticoagulada com varfarina por tromboembolismo pulmonar, apresentou-se com dor abdominal nos quadrantes inferiores. A TC abdominal revelou uma massa renal com hemorragia no espaço peri-renal. Foram ambas submetidas a embolização super-seletiva da artéria renal, com boa evolução posterior.
\end{abstract}

Palabras clave: síndrome de wunderlich, angiomiolipoma, femorragia renal espontánea

\section{INTRODUÇÃO}

A síndrome de Wunderlich, descrita inicialmente em 1856 por Carl Reinhold August Wunderlich como "apoplexia espontânea da capsula renal", define-se como hemorragia espontânea confinada ao espaço subcapsular e/ou perirrenal. A sua apresentação é heterogénea dependendo do grau e a duração da hemorragia. 1,23,4

Em doentes sintomáticos, a tríade clássica inclui dor lombar/ abdominal, massa palpável e choque hipovolémico. ${ }^{1,2,4}$ Outros sintomas podem estar presentes, nomeadamente náusea, vómitos, febre, anemia ou hematúria. ${ }^{1,4}$

As causas da síndrome de Wunderlich incluem neoplasias renais benignas e malignas, lesões vasculares como a poliarterite nodosa, infeções renais, nefrite, doenças hematológicas e alterações anatómicas como quistos e hidronefrose. ${ }^{2} \mathrm{~A}$ causa mais frequente é 0 angiomiolipoma (AML). ${ }^{1,2,3} 0 \mathrm{AML}$ é um tumor benigno composto por tecido adiposo, musculo liso e vasos com parede espessada. Devido à sua estrutura predispõe à formação de aneurismas e à sua rutura. ${ }^{1,2}$

Apresentamos dois casos de Síndrome de Wunderlich.

\section{CASO CLÍNICO}

\section{CASO 1}

Mulher de 38 anos, com esclerose tuberosa (linfangiomiomatose pulmonar e angiomiolipoma renal) com insuficiência respiratória crónica hipoxémica (sob oxigenoterapia de longa duração a 3 L/ minuto) recorreu ao Serviço de Urgência (SU) com dor no flanco esquerdo de início súbito, sem irradiação ou história de traumatismo, acompanhado de vómitos. Apresentava agravamento da hipoxemia ( $\mathrm{PaO}_{2} 46 \mathrm{mmHg}$ em ar ambiente) associado a hipotensão (Pressão arterial (PA) 84/58 mmHg). A hemoglobina era de $11.3 \mathrm{~g} / \mathrm{dL}$, leucócitos de $19.03 \times 10^{\circ} / \mathrm{L}$ sem trombocitopenia. A tomografia

\section{ABSTRACT}

Wunderlich syndrome is a rare disease characterized by spontaneous renal hemorrhage into the subcapsular and perirenal spaces. We report two cases of Wunderlich syndrome. A 38-year-old woman with tuberous sclerosis presented with abdominal pain and hypotension, perinephric hematoma was diagnosed by computerized tomography (CT) scan. A 86-year-old woman, treated with warfarin for pulmonary embolism, presented with abdominal pain in the lower quadrants. Abdominal CT scan revealed a renal mass with recent hemorrhage to perinephric space. Renal artery highly selective embolization was performed with good outcome in both cases.

Keywords: wunderlich syndrome, angiomyolipoma, spontaneous renal hemorrhage

computadorizada (TC) abdominal revelou uma densificação hemática extensa retroperitoneal esquerda, com epicentro peri-renal e um pequeno hemoperitoneu. Apresentava também uma massa renal exofítica esquerda no polo inferior com $80 \times 70 \times 62 \mathrm{~mm}$ de densidade heterogénea, hipervascular, correspondendo a um angiomiolipoma. (Figura 1AB) Após discussão clínica entre Urologia e Radiologia de intervenção a embolização foi adiada devia a estabilidade clínica após fluidoterapia.

Três dias após a admissão foi submetido embolização seletiva dos ramos inferiores da artéria renal esquerda, sem complicações imediatas. (Figura 1C) Cinco dias após o procedimento repetiu a TC abdominal mostrando redução do hematoma, uma área isquémica no polo inferior do rim esquerdo e reabsorção do hemoperitoneu. A doente não necessitou de suporte transfusional. A insuficiência respiratória melhorou durante 0 internamento com terapêutica broncodilatadora.

\section{CASO 2}

Mulher de 86 anos, sob terapêutica com varfarina por tromboembolismo pulmonar de alto risco de mortalidade 12 meses antes, com trombocitopenia não estudada, hipertensão arterial e doença renal crónica (Creatinina sérica basal 1,2 mg/dL) recorreu ao SU com dor abdominal no flanco/fossa ilíaca esquerda, sem história de traumatismo. Apresentava-se pálida, hidratada, com dor a palpação nos quadrantes inferiores do abdómen e múltiplas equimoses e petéquias nos membros inferiores. Na avaliação inicial a PA era de $112 / 90 \mathrm{mmHg}$, com agravamento progressivo para $70 / 32 \mathrm{mmHg}$ tendo desenvolvido taquicardia sinusal. Com a fluidoterapia instituída evoluiu favoravelmente revertendo a hipotensão. 0 estudo analítico revelou hemoglobina de $11.8 \mathrm{~g} / \mathrm{dL}$, leucócitos de $17.58 \mathrm{x}$ $10 \%$, trombocitopenia com plaquetas $<10000 / \mathrm{mm} 3$, lesão renal aguda (creatinina sérica de 2,17 mg/dL), lactato de $4.45 \mathrm{~mm} / \mathrm{L}$ e o International Normalized ratio de 3,14.

A ecografia abdominal revelou uma massa a envolver o rim esquerdo, melhor caracterizada por TC, mostrando um rim esquerdo aumentado 
Figura 1. (A) Corte transversal de TC abdominal com angiomiolipoma com hemorragia. (B) Corte coronal de TC abdominal revelando extensão de hemorragia. (C) Angiografia pós embolização de ramo inferior da artéria renal.
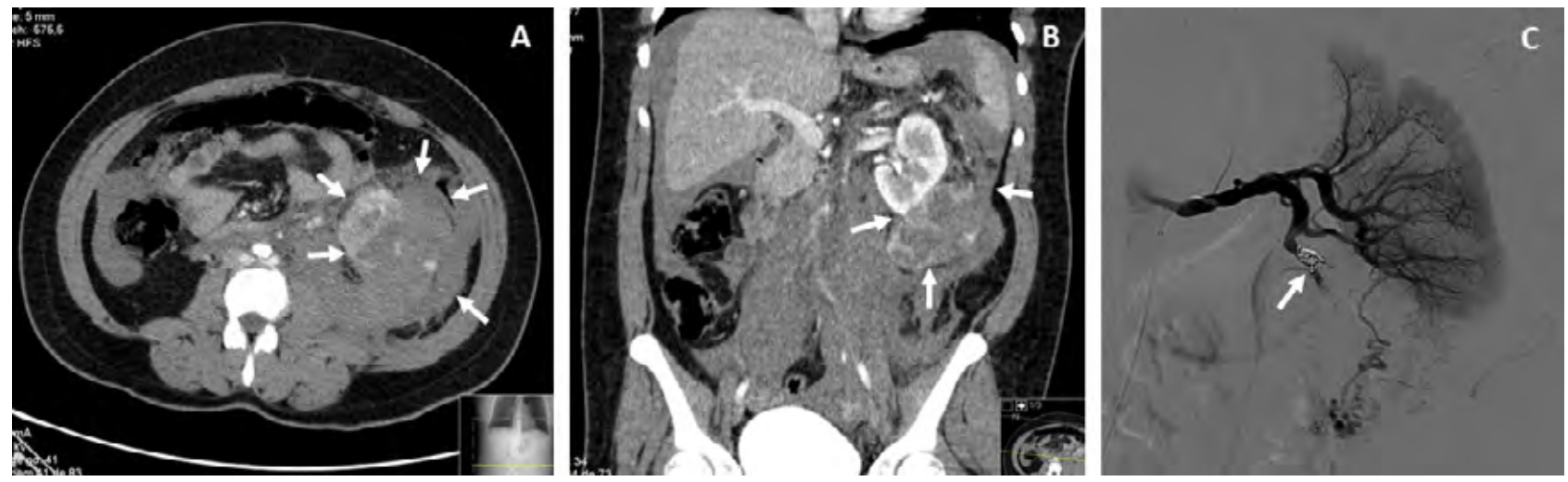

com massa exofítica nodular, realçada com contraste de $24 \times 22$ mm. Estava associada a uma extensa área hipodensa subcapsular, podendo corresponder a um hematoma com componente ativo. Este hematoma infiltrava os espaços peri e para-renal, adjacente à artéria e à veia renal. (Figura $2 \mathrm{AB}$ )

Foi medicada com vitamina Ke complexo protrombínico para reverter a anticoagulação. Realizou suporte transfusional com 6 unidades de plaquetas ainda no SU, tendo necessitado de 3 unidades de glóbulos vermelhos e outras 6 unidades de plaquetas durante 0 internamento. A doente iniciou tratamento com prednisolona $1 \mathrm{mg} /$ $\mathrm{Kg} /$ dia para púrpura trombocitopénica idiopática provável com meIhoria progressiva.

Foi submetida a embolização de um ramo da artéria renal esquerda com sucesso. A função renal estabilizou nos valores basais da doente. (Figura 2C) A doente foi orientada para consulta externa de urologia e hematologia. Durante o acompanhamento em consulta de urologia, manteve-se a abordagem conservadora, sendo a principal hipótese de diagnóstico 0 angiomiolipoma, atendendo à evolução e características em exames subsequentes.

\section{DISCUSSÃO}

A síndrome de Wunderliché uma doença rara e potencialmente fatal, que depende de um diagnóstico rápido e o seu correto tratamento para um bom resultado. 1,2,5

As neoplasias benignas são a causa mais comum, sendo 0 AML a mais frequente, com uma probabilidade de hemorragia até 50\% em tumores com mais de $40 \mathrm{~mm}$. 80\% dos AML são esporádicos sendo os restantes $20 \%$ relacionados com esclerose tuberosa. A neoplasia maligna mais comum é o carcinoma de células renais., ${ }^{1,2,5}$ Outras causas incluem outras neoplasias renais, infeção renal, vasculites, distúrbios da coagulação, entre outras. ${ }^{1-6}$ A síndrome de Wunderlich é a complicação mais letal do $\mathrm{AML},{ }^{1}$ relacionando-se com 0 seu tamanho. ${ }^{5}$

Nestes doentes a radiologia teve um papel fundamental no diagnóstico e tratamento. A TC abdominal é o melhor exame para o diagnóstico desta doença. ${ }^{2,6}$

0 tratamento inicial tem como objetivo a estabilidade hemodinâmica com fluidoterapia, transfusão de glóbulos vermelhos e correção dos distúrbios de coagulação. Em casos de hemorragia autolimitada a abordagem conservadora pode ser adotada. Nos casos de instabilidade hemodinâmica é necessário uma abordagem invasiva. ${ }^{6}$ Desde a primeira embolização de um angiomiolipoma renal em 1976 por Moorhead et al., a

Figura 2. (A) Corte transversal de TC abdominal de massa renal com hemorragia. (B) Corte coronal de TC abdominal revelando extensão de hemorragia. (C) Angiografia pós embolização de ramo inferior da artéria renal.
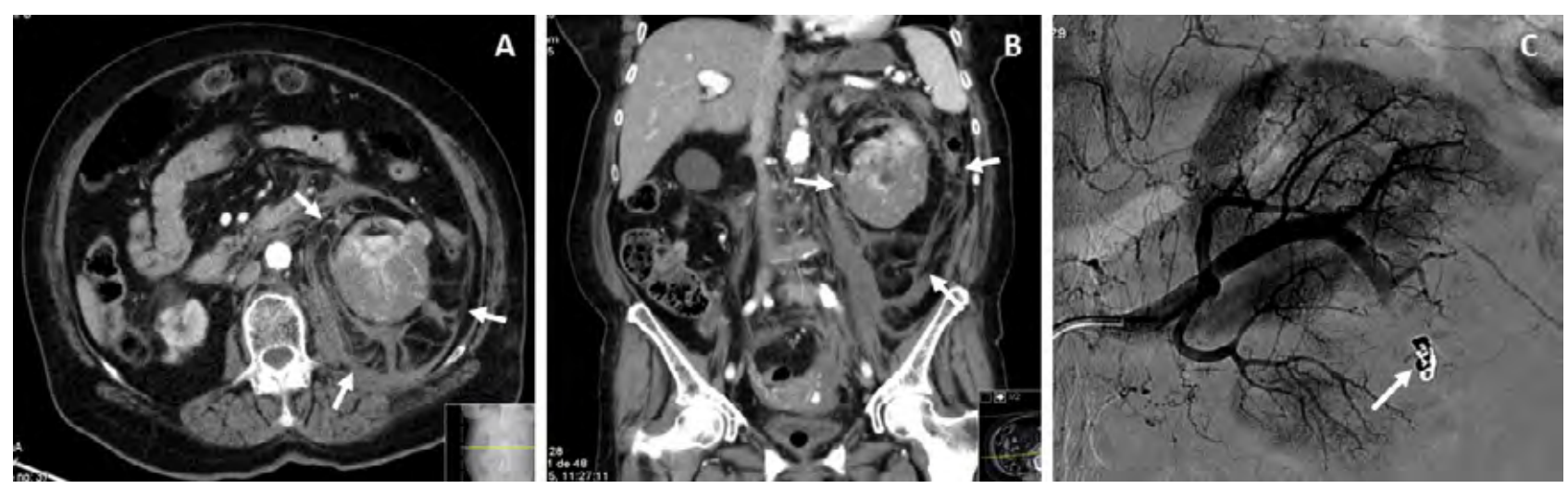
embolização selectiva dos AML com hemorragia tornou-se 0 tratamento recomendado, sendo seguro, eficiente e poupador de nefrónios. ${ }^{1,2,4,6,7}$ Quando a intervenção não é possível ou não alcança o resultado pretendido a nefrectomia (parcial ou total) é recomendada. A abordagem cirúrgica do hematoma pode agravar a hemorragia ${ }^{6,7} \mathrm{~A}$ abordagem por cirurgia aberta convencional é a mais frequente, sendo também possível a abordagem minimamente invasiva por laparoscopia. ${ }^{7}$

Na embolização seletiva dos ramos da artéria renal em doentes com AML a taxa de sucesso varia entre 80 e 90\%. Não é frequente 0 agravamento da função renal. A taxa de recidiva tumoral pode chegar a $43 \%$, sendo pior nos doentes com esclerose tuberosa. Estes doentes devem manter vigilância regular. ${ }^{8}$

Assim, os dois casos apresentados reforçam a necessidade de uma intervenção precoce na estabilidade do doente e colocam a embolização selectiva dos ramos da artéria renal como 0 tratamento de primeira linha no tratamento da síndrome de Wunderlich.

\section{BIBLIOGRAFIA}

1. Medda M, Picozzi SC, Bozzini G. Carmignani L. Wunderlich's syndrome and hemorrhagic shock. J Emerg Trauma Shock 2009; 2:203-5.

2. Parameswaran $B$, Khalid $M$, Malik N. Wunderlich syndrome following rupture of a renal angiomyolipoma. Ann Saudi Med 2006; 26 (4):310-2.

3. Mongha R, Bansal P, Dutta A, Das RK, Kundu AK. Wunderlich's syndrome with hepatic angiomyolipoma in tuberous sclerosis. Indian J Cancer 2008; 45 (2): 64-6.

4. Wang BH, Pureza V, Wang H, A tale of Wünderlich syndrome. J Surg Case Rep 2012; 2012(11):ris015.

5. Sotošek S, Marki囚D, Španjol J, Krpina K, Kneževi囚 S, Mari区i囚A. Bilateral Wünderlich Syndrome Caused by Spontaneous Rupture of Renal Angiomyolipomas. Case Rep Urol 2015;2015:316956, doi: 10.1155/2015/316956.

6. Rosenberg E, Yakov G, Maresky H, Neulander E. Spontaneous retroperitoneal hemorrhage (Wunderlich syndrome) due to renal cell carcinoma. Research 2014;1:622.

7. Bretterbauer K, Marki冈 D, Colleselli D, Hruby S, Magdy A, Janetschek G, et al. Laparoscopic Treatment of a Spontaneously Ruptured Kidney (Wunderlich Syndrome). Case Rep Urol. 2015;2015:701046. doi: 10.1155/2015/701046.

8. Sauk S, Zuckerman DA. Renal Artery Embolization. Semin Intervent Radiol 2011;28(4):396-406 